\title{
过渡金属催化的芳香化合物三氟甲基化研究进展
}

\author{
吕翠萍 $a$ 沈其龙 ${ }^{b}$ 刘 丹*, \\ ( ${ }^{a}$ 沈阳化工大学化学工程学院 沈阳 110142) \\ ${ }^{b}$ 中国科学院上海有机化学研究所 中国科学院有机氟化学重点实验室 上海 200032)
}

\begin{abstract}
摘要 向芳香化合物分子中引入三氟甲基在制药，农用化学品和有机材料的砌块合成中有重大的意义. 由于过渡金属 催化的反应反应条件温和，底物适用性广等优点，最近几年，逐渐被科学家应用到芳香化合物的三氟甲基化中，成为 追捧的热点. 综述了近几年在利用过渡金属对芳香化合物进行的三氟甲基化中取得的重大进展.

关键词 三氟甲基化; 过渡金属; 芳香化合物; 催化; 进展
\end{abstract}

\section{Transition Metal-Catalyzed Arene Trifluoromethylation}

\author{
Lü, Cuiping $^{a} \quad$ Shen, Qilong $^{b} \quad$ Liu, Dan ${ }^{*, a}$ \\ ( ${ }^{a}$ College of Chemical Engineering, Shenyang University of Chemical Technology, Shenyang 110142) \\ $\left({ }^{b}\right.$ Key Laboratory of Organofluorine Chemistry, Shanghai Institute of Organic Chemistry, \\ Chinese Academy of Sciences, Shanghai 200032)
}

\begin{abstract}
Incorporation of trifluoromethyl group into aromatic compounds is of great importance in the medicinal, agricultural, and material sciences. Owing to the mild reaction conditions and wide substrates scope, transition metal-catalyzed trifluoromethylation of arene substrates has attracted significant attention in the past few years. Herein, the recent progress in transition metal-catalyzed trifluoromethylation of arene substrates is surveyed and summarized.

Keywords trifluoromethylation; organometallic; arene substrates; catalysis; progress
\end{abstract}

芳香化合物分子中引入三氟甲基可以显著地改变 该化合物的物理、化学和生物性质, 如由于氟原子强吸 电性引起的分子的偶极距变化, 较强的碳一氟键(456 $486 \mathrm{~kJ} / \mathrm{mol}$ )又能增加化合物在生物体内的代谢适应 性 ${ }^{[1]}$. 同时三氟甲基的引入通常会增加分子的脂溶性, 从而有助药物分子在生物体内的吸收、传递和扩散. 因 而在制药、农药和有机材料的合成中得到越来越广泛的 应用 ${ }^{[2]}$.

化合物 $\mathbf{1}^{[3]}$ 是美国 Eli Lilly 公司开发的一种选择性 血清再吸收抑制剂 (S S R I) 型的抗抑郁药氟西汀 (Fluoxetine), 又称百优解. 自 1980 年上市以来, 氟西汀 一直是美国市场上处方量第三大的常用抗抑郁药物, 也 曾经是中国处方量最大的抗抑郁药物. 化合物 $\mathbf{2}^{[4]}$ 是由 拜耳公司开发的一类新型植物广谱杀菌剂. 化合物 $\mathbf{3}^{[5]}$ 是一种组织类胡萝卜素合成的除草剂达草减 (norflurazon), 在苯环的间位引入三氟甲基大大的增强
了其药效. 化合物 $\mathbf{4}^{[6]}$ ZLI-2857 是液晶显示屏的一种典 型的液晶材料(Scheme 1). 因此如何向芳香化合物分子 中引入三氟甲基已经成为有机化学界的一个研究热点.<smiles>CNCCC(Oc1ccc(C(F)(F)F)cc1)c1ccccc1</smiles><smiles>CO/N=C(/C(=O)OC)c1ccccc1CO/N=C(\C)c1cccc(C(F)(F)F)c1</smiles>

1<smiles>CNc1cnn(-c2cccc(C(F)(F)F)c2)c(=O)c1Cl</smiles>

3<smiles>CCCCC[C@H]1CC[C@H](c2ccc(-c3ccc(OCC)c(F)c3C(F)(F)Br)cc2)CC1</smiles>

4

Scheme 1

\footnotetext{
* E-mail: liudan20040318@163.com

Received October 15, 2011; revised February 10, 2012; published online March 22, 2012.

Project supported by the Shanghai Pujiang Program (No. 11PJ1412200).

上海市浦江人才计划(No. 11PJ1412200)资助项目.
} 
Grushin 等 ${ }^{[7]}$ 在 2011 年较全面地介绍了相关领域的化学 家们在芳烃三氟甲基化方面取得的重要进展. 本文综述 了近 5 年过渡金属催化的芳香化合物的三氟甲基化合成 中取得的一些重大进展.

\section{1 经典的芳环上三氟甲基引入方法}

\subsection{Swarts 反应}

三氟甲苯是在 19 世纪末由 Swarts $^{[8]}$ 首先合成的 (Scheme 2), 通过将甲苯甲基上的氢氯化后，再用氟原 子亲核取代氯从而生成三氟甲基. 但是由于该反应使用 等物质的量的催化剂, 产生大量的含氯的盐废弃物, 在 原子经济性和环境友好性上都存在很大的问题. 目前该 方法尚在工业上使用, 但是探索、发展新的, 环境友好 的三氟甲基化方法刻不容缓.

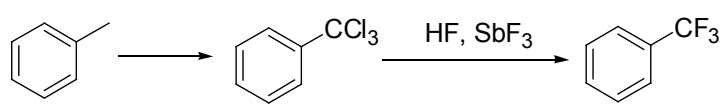

Scheme 2

\section{2 羒酸转化为三氟甲基}

Tullock 等 ${ }^{[9]}$ 在 20 世纪 70 年代通过一个两步反应将 脂肪族或芳香族羧酸的羧基在室温或更低的温度下高 产率地转化为三氟甲基(Scheme 3). 首先用较温和的氟 化剂如 $\alpha$-氟代烯胺或 DAST 将羟基转化成氟, 然后用 $\mathrm{SF}_{4}$ 将酰氟转化成三氟甲基. 而使用 $\mathrm{SF}_{4}$ 在无水 $\mathrm{HF}$ 中则 可一步将酸转化成三氟甲基.

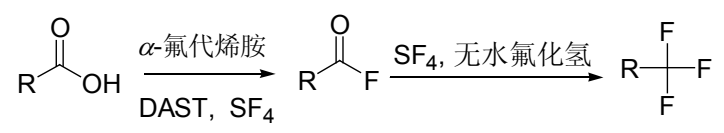

\section{Scheme 3}

\section{3 氧化脱硫氟化}

氧化脱硫氟化是在 20 世纪 70 年代发展起来的一种 合成三氟甲基取代的芳烃的新方法 ${ }^{[10]}$. 其基本概念就 是先将硫原子引入到有机分子中去作为氟的合成前体, 它再与一些氧化剂, 如 NBS, NIS, DBH, $\mathrm{Br}_{2}, \mathrm{SOCl}_{2}, \mathrm{~F}_{2}$, $\mathrm{IF}_{5}, p-\mathrm{MeC}_{6} \mathrm{H}_{4} \mathrm{IF}_{2}, \mathrm{NO}^{+} \mathrm{BF}_{4}^{-}$等在氟离子存在下进行氟 化反应, 实现三氟甲基化. 例如, 硫代黄原酸酯的硫代羰 基在较强的酸性介质中及用等物质的量的较强氧化剂 DBH 的条件下脱硫氟化, 以 $63 \%$ 的产率得到了芳环三 氟甲基化的产物(Eq. 1). 此方法的缺点是原子经济性不 强, 反应条件苛刻, 官能团的耐受性不强, 以及底物范 围受到限制.

\section{4 三氟甲基化自由基法}

芳香化合物与三氟甲基化自由基的反应发现地较

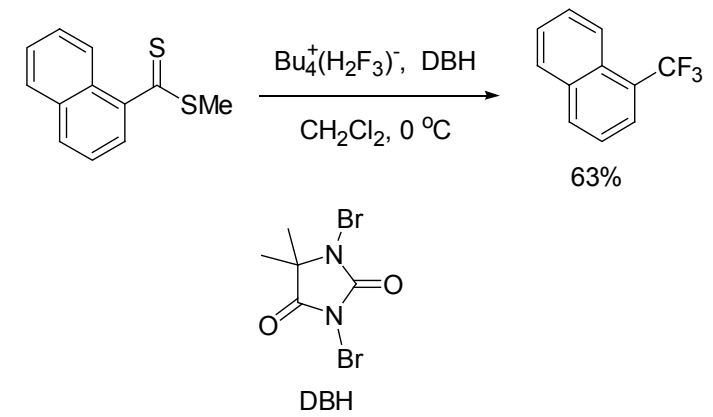

早 $^{[11]}$, 在 1960 年就有文献报道了过量的芳香化合物, 如苯, 甲苯，卤代苯，奈，苯甲腈，邻二苯甲酸䣶与三氟 甲基自由基的反应，以 $60 \% \sim 65 \%$ 的产率得到了相应的 三氟甲基化产物(Eq. 2). 但是这类反应容易产生二氟化 合物, 芳香底物也要大大过量, 此外, 反应的选择性也 不好.

$$
\mathrm{ArH}+2 \mathrm{R}_{\mathrm{fl}} \stackrel{250^{\circ} \mathrm{C}}{\longrightarrow} \mathrm{R} f \mathrm{Ar}+\mathrm{R}_{\mathrm{f}} \mathrm{H}+\mathrm{I}_{2}
$$

陈庆云等 ${ }^{[12]}$ 以 $\mathrm{DMF}$ 为溶剂, 紫外光照下使用 $\mathrm{CF}_{2} \mathrm{I}_{2}$ 与 $N$-甲基吡咯、2,4-二甲基吡咯、吲哚、咪唑、2-甲基 咪唑反应, 得到了含三氟甲基的芳杂环化合物(Scheme 4). 对于吡咯、吲哚、咪唑的三氟甲基化，均以 $2-\mathrm{CF}_{3}$ 为主. 通过机理的研究, 发现以上反应是一个光诱导电 子转移(PET)过程的自由基类型的三氟甲基化反应.

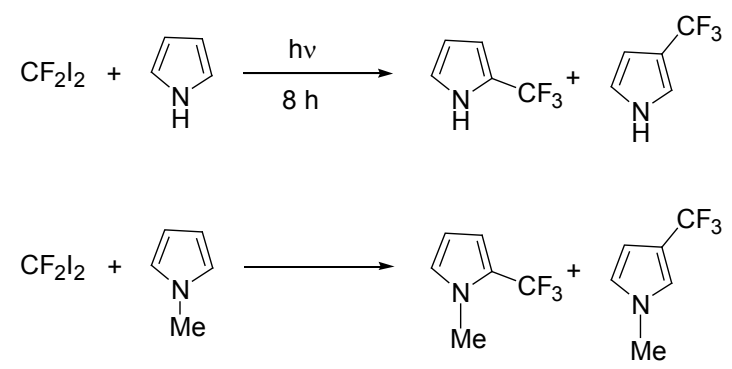

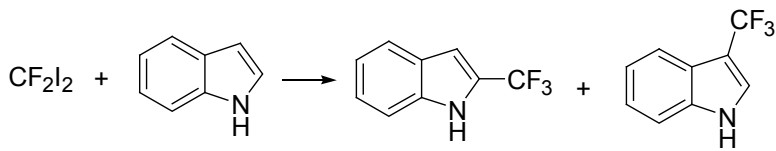

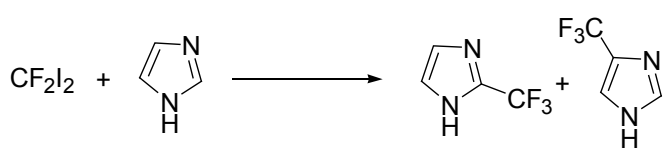

\section{Scheme 4}

相关的工作还有利用 Langlois 试剂加入催化量的铜 引发, 实现芳环上自由基类型的三氟甲基化 ${ }^{[13]}$ (Eq. 3).

与之类似的还有 Yamakawa 等 ${ }^{[14]}$ 研究的用催化量 的铁实现的芳环以及芳杂环化合物的三氟甲基化(Eq. 4), 但是产率并不高, 而且选择性也不好. 


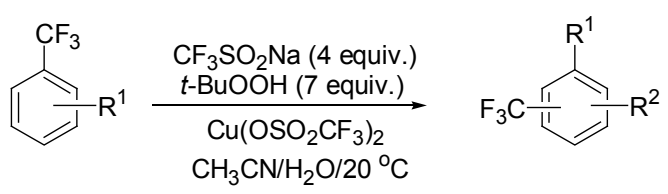<smiles>Nc1cccc2cccnc12</smiles>

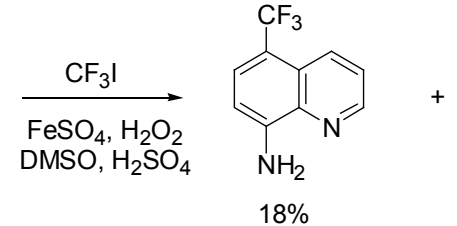<smiles>Nc1c(C(F)(F)F)ccc2cccnc12</smiles>

$9 \%$<smiles>Nc1c(C(F)(F)F)cc(C(F)(F)F)c2cccnc12</smiles>

最近, Baran 等 ${ }^{[15]}$ 发现以上反应不加金属催化剂, 也能很好地进行.

\section{5 利用三氟甲基铜试剂 $\left[\mathrm{CuCF}_{3}\right]$ 的芳烃三氟甲基化 反应}

铜催化的全氟碘代物与芳基碘反应制备全氟烷基 化的芳烃是 McLoughlin 和 Thrower 在一个专利中首次 报道的 ${ }^{[16]}$, 稍后 Kobayashi 和 Kumadaki ${ }^{[17]}$ 利用此方法合 成了一系列三氟甲基化的芳烃化合物.

在 20 世纪 80 年代, Yagupolskii ${ }^{[18]}$ 和 Burton 等 ${ }^{[19]}$ 通 过氟核磁观察到了三氟甲基铜物种 $\left[\mathrm{CuCF}_{3}\right]$, 虽然该试 剂是几个反应活性不同的含三氟甲基铜聚集体的混合 物. 他们利用不同的含氟原料合成了这个物种(Scheme 2)，并实现了一些芳基碘化合物的三氟甲基化.

随后上海有机所陈庆云小组 ${ }^{[20]}$ 发现 Chen 试剂与碘 化亚铜在 $60 \sim 80{ }^{\circ} \mathrm{C}$ 反应也生成了三氟甲基铜物种 $\left[\mathrm{CuCF}_{3}\right]$, 该铜试剂与芳基碘化合物反应高产率地合成 了三氟甲基化的芳烃(Scheme 5).

1991 年, Urata 和 Fuchikami ${ }^{[21]}$ 发现 Ruppert 试剂在 碱(如 TBAF, KF, $t$-BuOK)的作用下, 和碘化亚铜也生成 了三氟甲基铜物种 $\left[\mathrm{CuCF}_{3}\right]$ (Scheme 5).

\section{2 过渡金属参与的芳香化合物三氟甲基化}

以上介绍的主要是 1995 年以前实现的芳环上的三 氟甲基化反应. 最近 5 年, 越来越多的化学家将注意力 转移到这方面的研究上来, 使得过渡金属催化的芳环的 三氟甲基化成为一个研究热点. 其中用到的过渡金属催 化剂有 $\mathrm{Cu}^{[7]}, \mathrm{Pd}^{[7]}, \mathrm{Ru}^{[22]}, \mathrm{Ag}^{[23]}$. 本文就着重对近 5 年, 在过渡金属催化的芳香化合物的三氟甲基化中取得的 重要进展进行一下描述.

$$
\begin{aligned}
\mathrm{Hg}\left(\mathrm{CF}_{3}\right)_{2}+\mathrm{Cu} & \frac{\mathrm{NMP} \text { or DMAC }}{140{ }^{\circ} \mathrm{C}} \\
{\left[\mathrm{CuCF}_{3}\right] } & +\mathrm{Hg} \text { (Yagupolskii) }
\end{aligned}
$$$$
\begin{aligned}
& 2 \mathrm{M}+2 \mathrm{CF}_{2} \mathrm{X}_{2} \stackrel{\mathrm{DMF}}{\mathrm{r.t.}}\left[\mathrm{CF}_{3} \mathrm{MX}+\left(\mathrm{CF}_{3}\right)_{2} \mathrm{M}\right] \\
& \mathrm{M}=\mathrm{Cd}, \mathrm{Zn} \\
& \mathrm{X}=\mathrm{Br}, \mathrm{Cl}
\end{aligned}
$$

$$
\underset{\mathrm{Y}=\mathrm{I}, \mathrm{Br}, \mathrm{Cl}, \mathrm{CN}}{\stackrel{\mathrm{CN}}{\mathrm{C}}-80^{\circ} \mathrm{C} \sim \text { r.t. }}\left[\mathrm{CF}_{3} \mathrm{Cu}\right] \text { (Burton) }
$$$$
\mathrm{FO}_{2} \mathrm{SCF}_{2} \mathrm{CO}_{2} \mathrm{Me}+\mathrm{RX} \underset{\mathrm{DMF}, 60 \sim 80^{\circ} \mathrm{C}}{\stackrel{\mathrm{Cul}}{\longrightarrow}} \mathrm{RCF}_{3} \text { (Chen) }
$$$$
\mathrm{Arl}+\mathrm{CF}_{3} \mathrm{SiEt}_{3} \underset{\mathrm{KF}, \mathrm{Cul}(1 \sim 1.5 \text { equiv. })}{\longrightarrow} \mathrm{ArCF}_{3} \text { (Urata; Fuchikami) }
$$

\section{Scheme 5}

\section{1 铜试剂参与的芳环上的三氟甲基化}

\subsection{1 等物质的量的 $\mathrm{Cu}$ 试剂参与的芳环上的三氟甲} 基化

Vicic 小组 ${ }^{[24]}$ 成功地分离出第一个高活性的 N-杂环 卡宾(NHC)作为配体的三氟甲基铜试剂，该三氟甲基铜 试剂通过 X-ray 晶体衍射确定结构(Scheme 6), 此中间 体无论在室温下，还是在 $80{ }^{\circ} \mathrm{C}$ 下与芳基碘反应，都以 不低于 $90 \%$ 的产率得到了相应的三氟甲基化的产物.
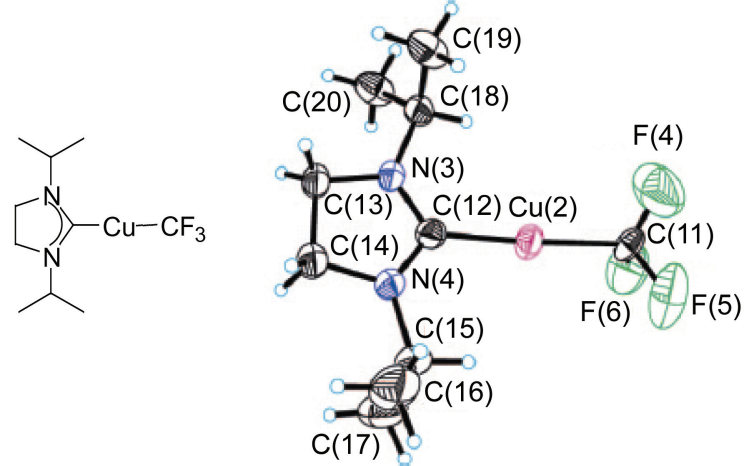

\section{Scheme 6}

Hartwig 小组 ${ }^{[25]}$ 用 1,10 -邻二氮菲作为配体也成功地 分离得到了三氟甲基铜试剂(Eq. 5). 这个中间体很稳定, 在室温和氮气保护下放置 1 个月未见分解.

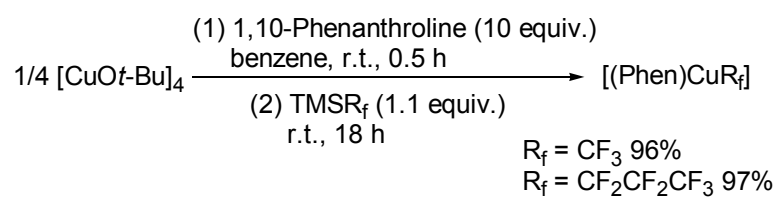

Hartwig 的三氟甲基铜试剂活性较高. 在室温到 50 ${ }^{\circ} \mathrm{C}$ 的条件下，该三氟甲基铜试剂和过量的芳基碘或芳基 
溴反应高产率地得到了相应的三氟甲基化的产物(Eq. 6). 尤其是对富电子的芳环，产率高达 86\% 96\%. 同 时该三氟甲基铜试剂对甲氧基、胺类、醛、醇羊基都有 很好的耐受性, 对于碱性的芳杂环也能忍受.

$$
\left[\left(\text { Phen) } \mathrm{CuCF}_{3}\right] \underset{\mathrm{Arl}(5.0 \text { equiv.) }}{\stackrel{\mathrm{DMF}, \text { r.t., } 18 \mathrm{~h}}{\mathrm{~h}}} \mathrm{ArCF}_{3}\right.
$$

同年, 翁志强等 ${ }^{[26]}$ 也分离到了 1,10 -邻二氮菲的衍 生物作为配体的三氟甲基铜试剂, 并通过 X-ray 晶体衍 射确定结构(Scheme 7). 只是该铜试剂活性稍低, $90{ }^{\circ} \mathrm{C}$ 时才与芳基碘化物反应以中等收率得到产物.

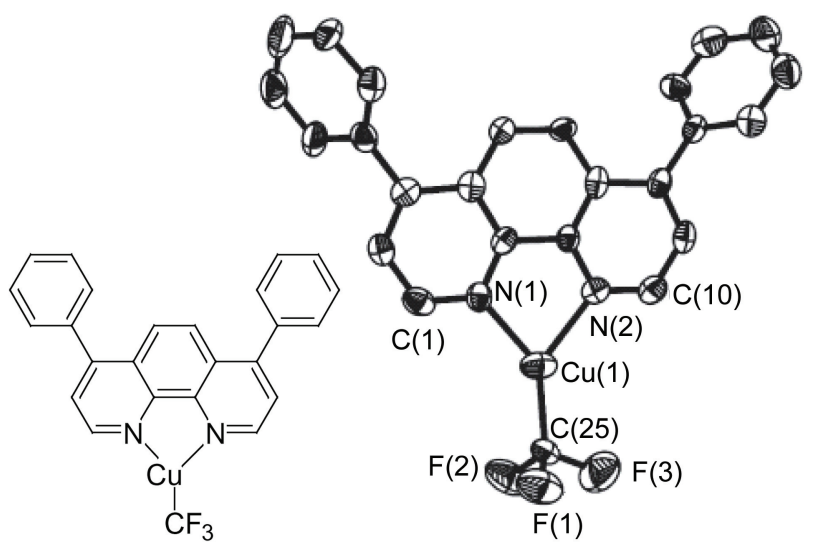

Scheme 7

Grushin 等 ${ }^{[27]}$ 也报道了三苯基膦作为配体的三氟甲 基铜试剂(Scheme 8), 该三氟甲基铜试剂与芳基碘化物 反应时活性不高, 需现场替换成联吡啶的配体才能得到 三氟甲基化的产物.

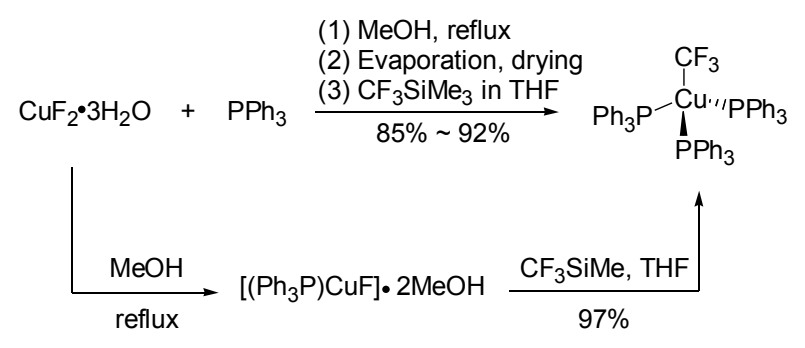

Scheme 8

最近, 肖吉昌小组 ${ }^{[28]}$ 首次用 $\mathrm{Cu}$ 还原锍鎓盐通过单 电子转移的方法实现三氟甲基化(Eq. 7). 他们发现芳杂 环碘化合物与二倍物等质量的量的锍鎓盐在 3 倍物质的 量的铜粉作用下 $60{ }^{\circ} \mathrm{C}$ 下搅拌 $11 \mathrm{~h}$, 得到了带供电子基 团的碘代芳杂环化合物三氟甲基化产物.

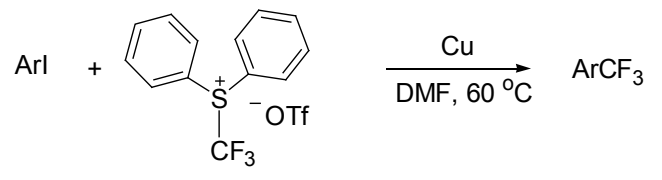

该反应可能的机理如 Scheme 9, 铜转移一个电子给 锍鎓盐，产生中间体，中间体又迅速地分解释放出三氟 甲基自由基，然后进一步生成三氟甲基铜中间体.
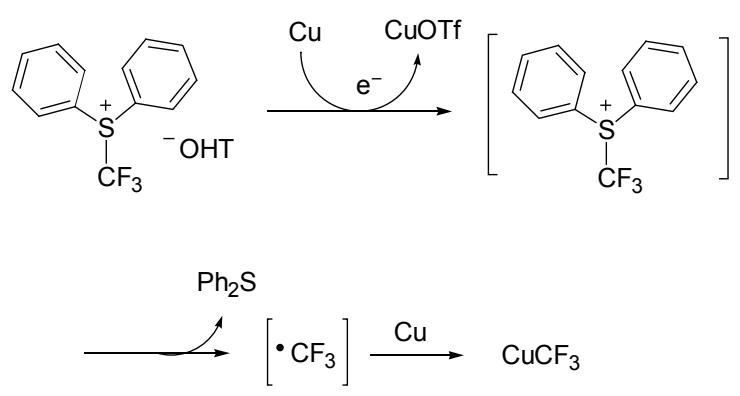

\section{Scheme 9}

2010 年, 卿凤领小组 ${ }^{[29]}$ 报道了第一例铜参与的氧 化条件下的芳基硼酸的三氟甲基化反应(Eq. 8). 该反应 反应条件比较温和 $\left(45 \sim 70{ }^{\circ} \mathrm{C}\right)$, 一些含硫含氧的官能 才都能忍受，芳基或烯基上含供电子基，吸电子基的底 物都能反应. 而且，羰基和溴取代的芳基硼酸能生成相 应的三氟甲基芳基化合物，羰基和溴在反应过程中保持 不变.

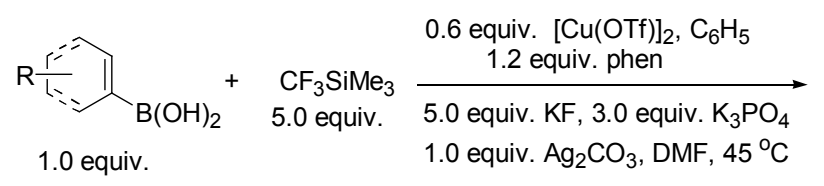

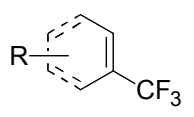

Buchwald 等 ${ }^{[30]}$ 报道了使用邻二氮杂菲作为配体, 对空气不敏感的醋酸铜作为催化剂, 利用氧气作为氧 化剂的芳基硼酸的三氟甲基化反应(Eq. 9). 该反应在室 温下以中等到良好的产率实现了取代苯环以及苯并芳 杂环的的三氟甲基化.

$$
\begin{aligned}
& \overbrace{}^{\mathrm{B}(\mathrm{OH})_{2}}+\mathrm{TMSCF}_{3} \frac{\mathrm{Cu}(\mathrm{OAc})_{2}}{\mathrm{Phenathroline}} \\
& \mathrm{CF}_{3}
\end{aligned}
$$

2.1.2 催化量的 $\mathrm{Cu}$ 试剂参与的芳环上的三氟甲基化 早在 1989 年, 陈庆云小组 ${ }^{[20]}$ 报道了碘化亚铜催化 的 Chen 试剂与芳基碘化物的三氟甲基化反应. 20 年后， Amii 小组 ${ }^{[31}$ 报道了首例通过配体稳定的催化量的碘化 亚铜催化的以亲核的 Ruppert 试剂作为三氟甲基来源的 
芳基碘化物的三氟甲基化(Eq. 10). 反应只适合缺电子 的碘苯, 对于富电子的碘苯并不反应, 对于电性中等的 四丁基碘苯也只有 50\%的产率.
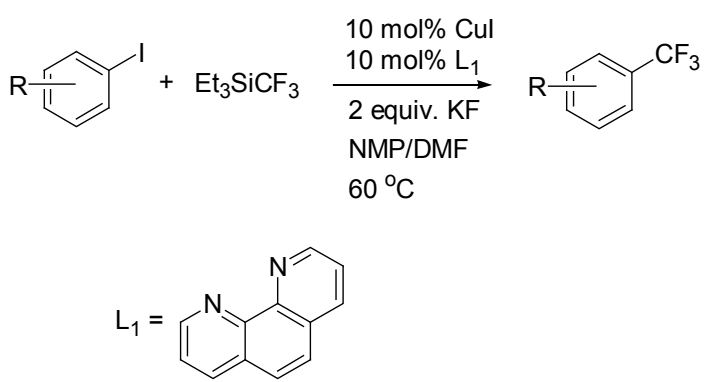

Burton 等 ${ }^{[19]}$ 使用二氟甲基及其衍生物 $\left(\mathrm{CF}_{2} \mathrm{Cl}_{2}\right.$, $\mathrm{CF}_{2} \mathrm{ClBr}, \mathrm{CF}_{2} \mathrm{Br}_{2}, \mathrm{CF}_{2} \mathrm{ClCO}_{2} \mathrm{X}$ 等) 以及 $\mathrm{Chen}$ 小组 ${ }^{[20]}$ 使用 陈氏试剂 $\left(\mathrm{FSO}_{2} \mathrm{CF}_{2} \mathrm{CO}_{2} \mathrm{Me}\right)$ 作为三氟甲基源合成了三氟 甲基铜试剂. 金属催化的芳环上的三氟甲基化中提供三 氟甲基源的试剂还有三氟甲基碘 $\left(\mathrm{CF}_{3} \mathrm{I}\right)$ 和三氟甲基溴

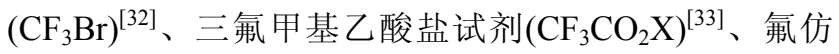
$\left(\mathrm{CF}_{3} \mathrm{H}\right)^{[34]}$ 以及 Ruppert-Prakash 试剂 ${ }^{[35]}$, 其中最常用的 是 Ruppert-Prakash 试剂. 但是由于 Ruppert-Prakash 试剂 沸点低 $\left(55{ }^{\circ} \mathrm{C}\right)$, 挥发性强, 而且使用时必须加碱活化, 因此寻找比 Ruppert-Prakash 试剂更稳定的三氟甲基试 剂就迫在眉捷. 最近 Gooßen 小组 ${ }^{[36]}$ 合成了一种高稳定 性的三氟甲基嗍酸三甲酯钾盐(Eq. 11). 该试剂在等物 质的量的 Ruppert-Prakash 试剂和硼酸三甲酯由氟化钾 作为碱反应而得, 产率将近 $100 \%$.

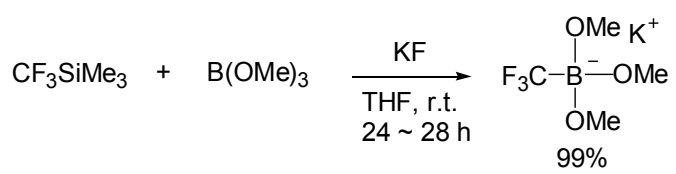

以它为三氟甲基源不需要加碱活化. 以铜作为催化 剂, 以优良的产率实现了一系列碘苯的三氟甲基化(Eq. 12). 缺电子、富电子的碘代芳香化合物均可反应, 并对 大多数官能团有一定的耐受性, 如酯、酰胺、胺. 杂环 化合物如碘代噻吩、碘代吡啶也可反应.

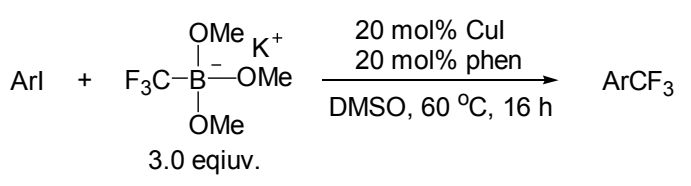

以上报道的铜催化的三氟甲基化反应都是以亲核 性的 Ruppert-Prakash 试作为氟源的, 一般须与较昂贵的 碘苯类化合物反应. 而利用亲电的三氟甲基试剂则可避 免使用碘苯类化合物. 最近，沈其龙小组 ${ }^{[37]}$ 报道了第一 例铜催化的芳基或烯基喼酸与 Togni 试剂发生的三氟甲
基化反应(Eq. 13). 用 Togni 试剂作为三氟甲基化试剂, 5 $\mathrm{mol} \%$ 的铜作为催化剂, 1,10-邻二氮杂菲作配体，两当量 的 $\mathrm{K}_{2} \mathrm{CO}_{3}$ 作碱, $35{ }^{\circ} \mathrm{C}$ 下反应 $14 \mathrm{~h}$, 可高产率地得到三氟 甲基化的产物.

此反应最大的优点是反应条件温和，底物范围广。 一般的芳基硼酸、苯并氮氧硫的杂环嗍酸、可烯醇化的 铜、酯、氨基甲酸酯以及氯代的芳香硼酸酯都以优良的 产率得到了相应三氟甲基化的产物. 烯基硼酸类化合物 也以良好的产率得到了三氟甲基化的产物.

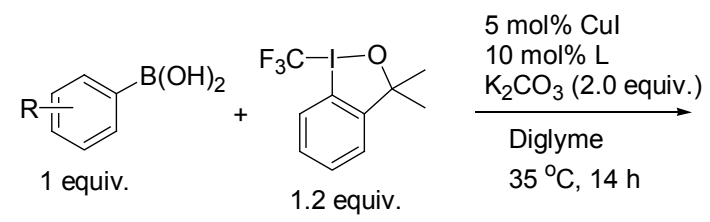

$$
\underbrace{C F_{3}}_{50 \% \sim 95 \%}
$$

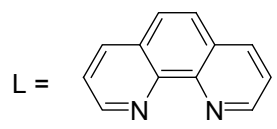

刘否小组 ${ }^{[38]}$ 和肖吉昌小组 ${ }^{[39]}$ 也报道了铜催化的芳 基嗍酸与亲电三氟甲基试剂 Umemoto 试剂或三氟甲基 锍鎓盐发生的三氟甲基化反应。这两个反应的产率中 等.

\section{$2.2 \mathrm{Pd}$ 参与的芳香化合物三氟甲基化}

\subsection{1 等物质的量的 $\mathrm{Pd}$ 参与的芳香化合物的三氟甲 基化}

Pd 催化的利用 Ruppert-Prakash 试作为氟源的三氟 甲基化反应迄今为止只有一例. 其主要问题是三氟甲基 转金属到钯上后生成的 $\mathrm{Pd}-\mathrm{CF}_{3}$ 键能比较大，还原消除 比较难. 最近几年的研究发现, 利用配体的电子性和位 阻效应可以实现芳基和三氟甲基从二价钯上的还原消 除反应.

Grushin 小组 ${ }^{[40]}$ 用 $[(\mathrm{dppe}) \mathrm{Pd}(\mathrm{I}) \mathrm{Ph}]$ 在过量的 $\mathrm{Me}_{3} \mathrm{Si}$ $\mathrm{CF}_{3} / \mathrm{CsF}$ 中以 $69 \%$ 的产率合成了 $\left[(\mathrm{dppe}) \mathrm{Pd}\left(\mathrm{CF}_{3}\right) \mathrm{Ph}\right.$. 然 而 $\left[(\mathrm{dppe}) \mathrm{PdPh}\left(\mathrm{CF}_{3}\right)\right]$ 的还原消除反应很慢，只有在 $\mathrm{PhI}$ 存在下, $145{ }^{\circ} \mathrm{C}$ 的高温下反应 $64 \mathrm{~h}$, 才能以 $60 \%$ 的产率 得到 $\mathrm{Ph}-\mathrm{CF}_{3}$ (Eq. 14). 反应的机理尚不清楚.

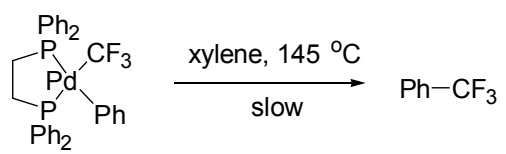

Grushin 小组 ${ }^{[41]}$ 又合成了 Xantphos 为配体的络合物 
[XantphosPd $\left.(\mathrm{Ph})\left(\mathrm{CF}_{3}\right)\right]$. 此钯络合物在加热到 $80{ }^{\circ} \mathrm{C}$ 的 条件下反应 $3 \mathrm{~h}$, 以 $100 \%$ 的产率生成了 $\mathrm{Ar}-\mathrm{CF}_{3}$, 这是 首例报道的高产率的生成 $\mathrm{ArCF}_{3}$ 的二价钯上的还原消除 反应(Eq. 15).

$$
\left[(\text { Xantphos }) \mathrm{PdCF}_{3} \mathrm{Ph}\right]+\text { Xantphos } \frac{\mathrm{C}_{6} \mathrm{H}_{6}, 80^{\circ} \mathrm{C}, 3 \mathrm{~h}}{100 \%} \mathrm{PhCF}_{3}+
$$$$
(\text { Xantphos })_{2} \mathrm{Pd}
$$<smiles>CC1(C)c2cccc(-c3ccccc3)c2Oc2c(-c3ccccc3)cccc21</smiles>

以上描述的含三氟甲基的二价钯络合物的还原消 除反应基本都是通过配体的立体效应和电子效应的调 节实现芳基和三氟甲基从 $\mathrm{Pd}^{\mathrm{II}}$ 上还原消除, 得到 $\mathrm{Ar}-$ $\mathrm{CF}_{3}$. 那么通过改变 $\mathrm{Pd}$ 的氧化态, 而不是金属中心的配 体, 是否可以使得 $\mathrm{Ar}-\mathrm{CF}_{3}$ 的还原消除反应在动力学和 热力学上更容易?

Sanford 小组 ${ }^{[42]}$ 以联吡啶为配体, 成功地合成了含 三氟甲基的四价钯的络合物(Eq. 16), 该络合物的结构 由 X-ray 单晶衍射确证. 将该四价钯络合物加热到 80 ${ }^{\circ} \mathrm{C}$ 反应 $3 \mathrm{~h}$, 以 $70 \%$ 的产率得到了三氟甲基化的产物.

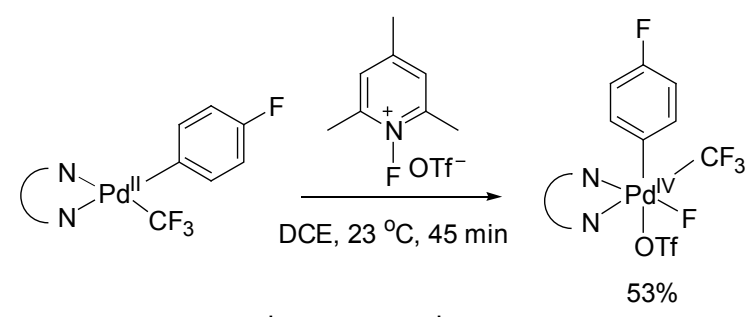

$|\mathrm{N}-\mathrm{N}=\mathrm{dtbpy}|$

通过对还原消除过程机理的研究, 发现经历了一个 $\mathrm{TfO}^{-}$的解离平衡, 接着发生了 $\mathrm{Ar}-\mathrm{CF}_{3}$ 的偶联反应. 通 过 DFT 计算也表明三氟甲基作为一个亲电试剂, 芳基 配体作为亲核试剂与其发生偶联.

通过以上的机理, 用 tmeda ( $N, N, N, N$-tetramethylethylenediamine)代替 dtbpy 作为配体, 在室温下实现了 氧化的三氟甲基化反应(Eq. 17).

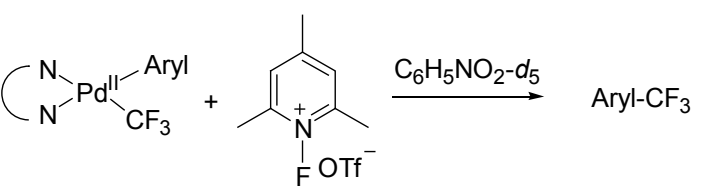

$$
\begin{aligned}
& {[\mathrm{N}-\mathrm{N}=\mathrm{dtbpy}]}
\end{aligned}
$$

2.2.2 催化量的 $\mathrm{Pd}$ 参与的芳香化合物三氟甲基化 Buchwald 小组 ${ }^{[43]}$ 以芳基氯作为底物的三氟甲基化
反应是氟化学领域里的一个突破性的进展. Buchwald 小 组首先研究了等物质的量的三配位的二价钯络合物的 还原消除反应. 他们发现当以 BrettPhos 作为配体时, 60 ${ }^{\circ} \mathrm{C}$ 下反应 $20 \mathrm{~h}$ 即可以 $28 \%$ 产率得到还原消除产物.

随后他们把等物质的量的反应发展成催化反应. 该 方法用 $\left[\{\mathrm{Pd}(\mathrm{allyl}) \mathrm{Cl}\}_{2}\right]$ 作为钯源, Brettphos 作为配体, $\mathrm{TESCF}_{3} / \mathrm{CsF}$ 作为三氟甲基源, 1,4 -二氧六环溶液作为溶 剂, 和芳基氯化物在 $120 \sim 140{ }^{\circ} \mathrm{C}$ 下搅拌 $6 \sim 20 \mathrm{~h}$, 以 $80 \%$ 的产率成功地实现了吲哚类、咔唑类、喹啉和苯并 呋喃的三氟甲基化(Eq. 18). 芳环上有供电子基团、吸电 子基团的芳基氯化合物都可以反应，但由于 $\mathrm{CF}_{3}^{-}$会发 生质子化，对于醛或酮，含有未被保护羟基、胺基等基 团的底物并不适用.

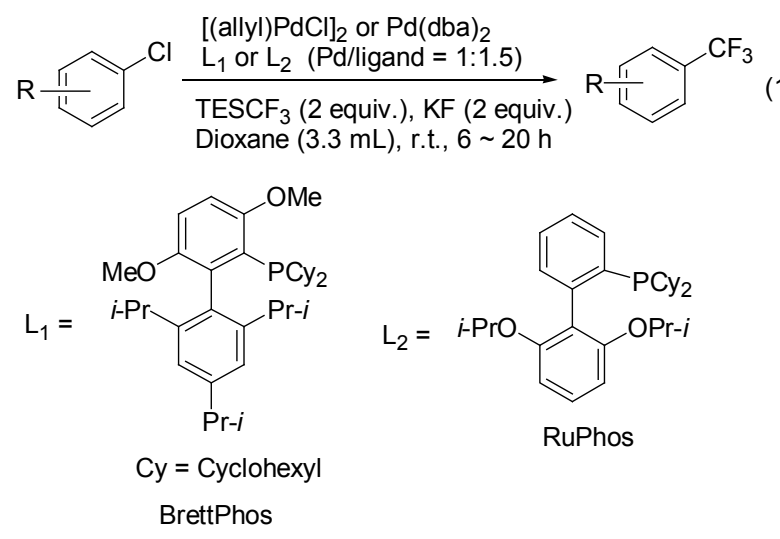

$\mathrm{C}-\mathrm{H}$ 键活化官能团化是当前有机化学研究的热点 领域之一. 最近, $\mathrm{Yu}$ 等 ${ }^{[44]}$ 报道了钯催化的通过 $\mathrm{C}-\mathrm{H}$ 键 活化而实现的芳基的三氟甲基化反应(Eq. 19). 该反应 用 $10 \%$ 的 $\mathrm{Pd}(\mathrm{OAc})_{2}$ 作钯源，1,2-二氯乙烷和三氟乙酸作 溶剂, 然后用等物质的量的 Umemoto 试剂实现芳基诱 导基团邻位的三氟甲基化. 反应体系中 TFA 是反应发 生的关键, 用 $\mathrm{Cu}(\mathrm{OAc})_{2}$ 作为氧化剂可以大大提高催化 反应的转化率. 当底物有给电子取代基如甲基、甲氧基 或被氯取代时，有很好的反应性，但当有吸电子基如羰 基、酯基、硝基时，目标产物的产率不足 $20 \%$. 同时荎 环也可以反应，诱导基团可以为是吡啶、嘧啶、咪唑、 噻唑.

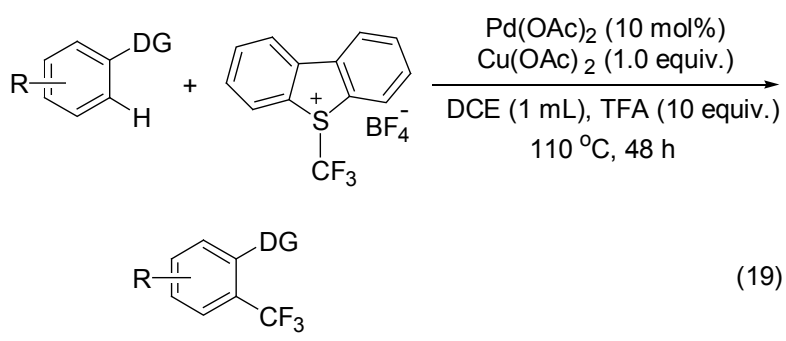

最近, 刘国生小组 ${ }^{[4]}$ 通过 Pd 催化的氧化加成的三 氟甲基化, 实现了吲哚环上的三氟甲基化(Eq. 20). 


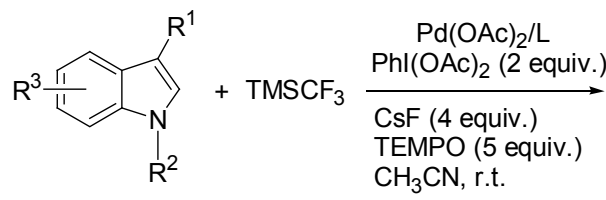

$\underbrace{\mathrm{R}^{1}}_{\mathrm{R}^{2}}$

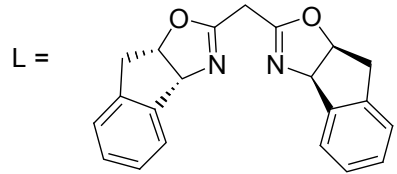

\section{3 其它金属参与的芳环上的三氟甲基化}

除了以上常用的 $\mathrm{Cu}$ 和 $\mathrm{Pd}$ 作为金属催化剂. 化学家 们还尝试了其他的金属催化的此类反应, 其中, 已经取 得成功的金属有 $\mathrm{Ru}^{[22]}, \mathrm{Ag}^{[23]}$.

1994 年, Kamigata 等 $^{[22]}$ 用 Langlois 试剂, 在 2 $\mathrm{mol} \% \mathrm{RuCl}_{2}\left(\mathrm{PPh}_{3}\right)_{3}$ 催化下, $120{ }^{\circ} \mathrm{C}$ 的条件下反应 $18 \mathrm{~h}$, 以中等产率得到了相应的三氟甲基化产物(Eq. 21). 但 是对于有取代基的苯环, 三氟甲基化产物的选择性并不 好.

$$
+\mathrm{CF}_{3} \mathrm{SO}_{2} \mathrm{Cl} \frac{2 \mathrm{~mol} \% \mathrm{RuCl}_{2}\left(\mathrm{PPh}_{3}\right)_{3}}{120^{\circ} \mathrm{C}, 18 \mathrm{~h}} \stackrel{\mathrm{l}}{\mathrm{I}} \mathrm{CF}_{3}
$$

Sandford 等 ${ }^{[23]}$ 在今年实现的 $\mathrm{Ag}$ 参与的芳环上的三 氟甲基化(Eq. 22).

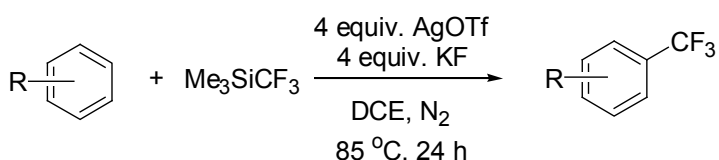

\section{4 结论和展望}

芳环上的三氟甲基化在制药和合成材料方面的重 大应用价值使它的研究成为一个热点, 在过去的几年 里，过度金属参与或催化的卤代芳烃和芳基硼酸的三氟 甲基化和钯催化的芳烃 $\mathrm{C}-\mathrm{H}$ 键活化三氟甲基化反应已 经取得了显著的突破. 接下来的发展方向应是寻找新的 三氟甲基化试剂, 更合适的三氟甲基化催化剂, 寻找温 和的反应条件以及扩大反应底物范围.

\section{References}

[1] Purser, S.; Moore, P. R.; Swallow, S.; Gouverneur, V. Chem. Soc. Rev. 2008, 37, 320.

[2] (a) Kirk, K. L. Org. Process Res. Dev. 2008, 12, 305. (b) Kirsch, P.; Bremer, M. Angew. Chem., Int. Ed. 2000, 39, 4216.

[3] Wong. D. T.; Perry, K. W.; Bymaster, F. P. Nat. Rev. Drug Discovery 2005, 4, 764 .

[4] Margot, P.; Huggenberger, F.; Amrein, J.; Weiss, B. BCPC Conf.-Pests. Dis. 1998, 2, 375

[5] Jeschke, P.; Jeschke, P. ChemBioChem 2004, 5, 570.

[6] Reiffenrath, V.; Krause, J.; Plach, H. J.; Weber, G. Liq. Cryst. $\mathbf{1 9 8 9}, 5,159$

[7] Tomashenko, O. A.; Grushin, V. V. Chem. Rev. 2011, 111, 4475.

[8] Swarts, F. Bull. Acad. R. Med. Belg. 1892, 24, 415.

[9] (a) Boswell, G. A.; Pipka, Jr. W. C.; Schribner, R. M.; Tullock, C. W. Org. React. 1972, 21, 1

(b) Wang, C.-L. J. Org. React. 1985, 34, 319

[10] Kirsch, P. Modern Fluoroorganic Chemistry, Wiley-VCH, Weinheim, Germany, 2004, p. 68.

[11] Tiers, G. V. D. J. Am. Chem. Soc. 1960, 82, 5513

[12] Chen, Q.-Y. Li, Z.-T. J. Chem. Soc. 1993, 6, 645.

[13] Langlois, B.; Laurent, E.; Roidot, N. Tetrahedron Lett. 1991, 32, 7525 .

[14] Kino, T.; Nagase, Y.; Ohtsuka, Y.; Yamamoto, K.; Uraguchi, D.; Tokuhisa, K.; Yamakawa, T. J. Fluorine Chem. 2010, 131, 98.

[15] Ji, Y.; Brueckl, T.; Baxter, R. D.; Fujiwara, Y.; Seiple, I. B.; Su, S.; Blackmond, D. G.; Baran, P. S. Proc. Natl. Acad. Sci. U. S. A. 2011, 108, 14411 .

[16] McLoughlin, V. C. R.; Thrower, J. US 3408411, 1968 [Chem. Abstr. 1968, 68, 11002].

[17] Kobayashi, Y.; Kumadaki, I. Tetrahedron Lett. 1969, 4095.

[18] Kondratenko, N. V.; Vechirko, E. P.; Yagupolskii, L. M. Synthesis, 1980932.

[19] (a) Burton, D. J.; Wiemers, D. M. J. Am. Chem. Soc. 1985, 107, 5014.

(b) Burton, D. J.; Wiemers, D. M. J. Am. Chem. Soc. 1986, 108, 832.

[20] Chen, Q.-Y.; Wu, S.-W. Chem. Commun. 1989, 705.

[21] Urata, H.; Fuchikami, T. Tetrahedron Lett. 1991, 32, 91.

[22] Kamigata, N.; Ohtsuka, T.; Fukushima, T; Yoshida, M.; Shimizu, T. Perkin Ttrans. 1994, 1339.

[23] Ye, Y.; Lee, S. H.; Sanford, M. S. Org. Lett. 2011, 13, 5464.

[24] (a) Dubinina, G. G.; Furutachi, H.; Vicic, D. A. J. Am. Chem. Soc. 2008, 130,8600

(b) Dubinina, G. G.; Ogikubo, J.; Vicic, D. A. Organometallics 2008, 27, 6233 .

[25] Hartwig. J. F. Angew. Chem., Int. Ed. 2011, 50, 3793.

[26] Weng, Z.-Q.; Lee, R.; Jia, W.-G.; Yuan, Y.-F.; Wang, W.-F.; Huang, K.-W. Organometallics 2011, 30, 3229.

[27] Olesya, A.; Tomashenko, E. C.; Escudero, A.; Belmonte, M. M.; Grushin, V. V. Angew. Chem., Int. Ed. 2011, 50, 7655.

[28] Zhang, C.-P.; Wang, Z.-L.; Chen, Q.-Y.; Zhang, C.-T.; Gu, Y.-C.; Xiao, J.-C. Angew. Chem., Int. Ed. 2011, 123, 1936.

[29] Chu, L.-L.; Qing, F.-L. Org. Lett. 2010, 12, 5060.

[30] Senecal, T. D.; Parsons, A. T.; Buchwald, S. L. J. Org. Chem. 2011, 76, 1174.

[31] Oishi, M.; Kondo, H.; Amii, H. Chem. Commun. 2009, 1909.

[32] (a) Hughes, R. P. J. Organomet. Chem. 1990, 31, 183.

(b) Morrison, J. A. J. Organomet. Chem. 1993, 35, 211.

[33] (a) Matsui, K.; Tobita, E.; Ando, M.; Kondo, K. Chem. Lett. 1981, 1719.

(b) Langlois, B. R.; Roques, N. J. Fluorine Chem. 2007, 128, 1318.

[34] (a) Folleas, B.; Marek, I.; Normant, J.-F.; Saint-Jalmes, L. Tetrahedron 2000, 56, 275.

(b) Prakash, G. K. S.; Hu, J.; Olah, G. A. Org. Lett. 2003, 5, 3253.

[35] (a) Prakash, G. K. S.; Yudin, A. K.; Deffieux, D.; Olah, G. A. 
Synlett 1996, 151.

(b) Huang, D.; Caulton, K. G. J. Am. Chem. Soc. 1997, 119, 3185.

[36] Knauber, T.; Arikan, F.; Röschenthaler, G.; Gooßen, L. J. Chem. Eur. J. 2011, 17, 2689.

[37] Liu, T.-F.; Shen, Q. Org. Lett. 2011, 13, 2342.

[38] Xu, J.; Luo, D.-F.; Xiao, B.; Liu, Z.-J.; Gong, T.-J.; Fu, Y.; Liu, L. Chem. Commun. 2011, 47, 4300.

[39] Zhang, C.-P.; Cai, J.; Zhou, C.-B.; Wang, X.-P.; Zheng, X.; Gu, Y.-C.; Xiao, J.-C. Chem. Commun, 2011, 47, 9516.

[40] Grushin, V. V.; Marshall, W. J. J. Am. Chem. Soc. 2006, 128, 4632.
[41] Grushin, V. V.; Marshall, W. J. J. Am. Chem. Soc. 2006, 128, 12644.

[42] Nicholas, D.; Ball, J.; Gary, B.; Ye, Y.; Sanford, M. S. J. Am. Chem. Soc. 2011, 133, 7577.

[43] Cho, E. J.; Senecal, T. D.; Kinzel, T.; Zhang, Y.; Watson, D. A.; Buchwald, S. L. Science 2010, 328, 1679.

[44] Wang, X.; Truesdale, L.; Yu, J. Q. J. Am. Chem. Soc. 2010, 132, 3648.

[45] Mu, X.; Chen, S.-J.; Zhen, X.-L.; Liu, G.-S. Chem. Eur. J. 2011, 17,6039 .

(Qin, X.) 